\title{
Keratin 36, a specific marker of tongue filiform papillae, is downregulated in squamous cell carcinoma of the mobile tongue
}

\author{
VERONIKA BRYCHTOVA ${ }^{1}$, PHILIP J. COATES ${ }^{1}$, VACLAV HRABAL ${ }^{1}$, LINDA BOLDRUP ${ }^{2}$, \\ PAVEL FABIAN $^{3}$, BORIVOJ VOJTESEK ${ }^{1}$, NICOLA SGARAMELLA ${ }^{2}$ and KARIN NYLANDER ${ }^{2}$ \\ ${ }^{1}$ Regional Centre for Applied Molecular Oncology, Masaryk Memorial Cancer Institute, CZ-656 53 Brno, \\ Czech Republic; ${ }^{2}$ Department of Medical Biosciences, Umea University, SE-901 85 Umea, Sweden; \\ ${ }^{3}$ Department of Pathology, Masaryk Memorial Cancer Institute, CZ-656 53 Brno, Czech Republic
}

Received October 10, 2019; Accepted February 5, 2020

DOI: $10.3892 / \mathrm{mco} .2020 .2005$

\begin{abstract}
Human keratin 36 (K36) is a member of the hair keratin family and is a marker of hair cortex differentiation. The human KRT36 gene is located on the long arm of chromosome 17 and belongs to the cluster of structurally unrelated acidic hair keratins. Recently, it has been reported that KRT36 mRNA is specifically expressed in normal tongue epithelium and downregulated in squamous cell carcinomas of the mobile tongue. Furthermore, KRT36 levels have been reported to be downregulated in clinically normal mobile tongue tissue that is adjacent to tumours, suggesting it could be a marker of pre-neoplastic changes. However, the exact role and the potential role of K36 in tongue tumour formation remains unclear. The aim of the present study was to investigate expression of $\mathrm{K} 36$ in a series of squamous cell carcinomas of the mobile tongue, normal mobile tongue and a small panel of other human tissues (normal tissue from the appendix, cervix, hair, lip, mamilla, nail, oesophagus, skin, thymus and vagina) and selected cancer tissue (cervical cancer, melanoma and basal cell carcinoma). Affinity purified polyclonal antibodies against K36 were generated and used for immunohistochemical analysis. The results revealed that in the normal tongue, K36 was detected specifically in the filiform papillae of the dorsal surface of the tongue. Additionally, none of the tongue cancer tissue samples were K36-positive. Immunostaining also revealed that $\mathrm{K} 36$ was expressed in nail beds, Hassal's corpuscles in the thymus and the hair cortex.
\end{abstract}

Correspondence to: Professor Karin Nylander, Department of Medical Biosciences, Umea University, Building 6M, 2nd floor, SE-901 85 Umea, Sweden

E-mail: karin.nylander@umu.se

Dr Veronika Brychtova, Regional Centre for Applied Molecular Oncology, Masaryk Memorial Cancer Institute, Zluty kopec 7, CZ-656 53 Brno, Czech Republic

E-mail: vebrychtova@mou.cz

Key words: keratin 36, mobile tongue, squamous cell carcinoma, thymus, nail
However, K36 was not expressed in the squamous epithelia of the skin, cervix and oesophagus, and the squamous cells of cervical carcinomas, basal cell carcinoma or melanoma. The present data indicated that K36 may be inactivated in tumours of the tongue. However, whether this is part of the tumoural process or if it is an effect of the tumour itself remains to be elucidated.

\section{Introduction}

Keratins comprise a large group of structural proteins of the intermediate filament protein family and are found in all epithelial cells. Expression of individual keratins is cell and tissue specific and reflects type of epithelium as well as state of differentiation of the epithelial cells. Keratins possess viscoelastic properties and thus provide cells with mechanical stability and help to maintain cellular integrity. At the same time keratins act in intracellular signalling pathways during wound healing and stress responses (1-3).

Based on gene structure, cysteine/proline content of the carboxy-terminal region and specific expression patterns, keratins can be classified as 'soft' cytokeratins expressed in various types of epithelia or 'hair' (trychocytic) keratins that are components of epithelial appendages. The biochemical properties of both groups classify keratins as type I (acidic) or type II (basic to neutral isoelectric point). All keratins share a tripartite domain structure with a central self-assembling $\alpha$-helical rod domain that allows them to form heterodimeric complexes containing a type I and II keratin in equimolar amounts. These subunits form the basis of the intermediate filament network. Specific keratin pairs tend to be co-expressed and serve as markers of epithelial differentiation (4).

Mammalian hair keratins are involved in the formation of hard keratinizing structures such as the hair-forming compartment of the hair follicle and distinct nail compartments. Although their prominent expression is in hair, dorsal tongue epithelium as well as other soft human epithelia also express hair type keratins (4-8). Human hair keratins comprise nine type I members (K31, K32, K33a, K33b, K34, K35, K36, K37 and K38) and six type II members (K81, K82, K83, K84, K85 and K86). In situ hybridization and immunohistochemical studies have shown each member to have a specific location 
within the hair follicle or tongue epithelium corresponding to the stage of cellular differentiation $(2,9,10)$.

Human keratin 36 [K36, previously termed Ha6 and $\mathrm{Ka} 31$ (2)] is classified as a type I hair keratin, which presumably partners with an unknown type II keratin. K36 has been described as a marker of advanced stage of differentiation in upper hair cortex, although it does not represent the major hair keratin (9). The human KRT36 gene that encodes K36 is located within a type-specific cluster on chromosome 17q12-q21 and belongs to the structurally unrelated group $\mathrm{C}$ of acidic hair keratins (9).

The group of oral squamous cell carcinoma (OSCC) exerts the eighth most common cancer in the world, with a 5-year survival $<60 \%$ (11). Within this group tumours are most commonly located in the tongue (SCCOT), and differences between these SCCOTs and SCCs in other intra-oral sites have been shown $(12,13)$. Worldwide there is an increasing incidence of SCCOT, particularly among young people ( $<45$ years) and in many parts of the World especially women (11).

In a patient with OSCC not only the tumour but also the surrounding tissue shows neoplastic changes, a phenomenon called field cancerization (14). Genetically changed fields can in cases of OSCC be found within a distance of $7 \mathrm{~cm}$ from the tumour (14), which in the case of SCCOT means that the whole tongue can be affected.

Looking at the adjacent clinically tumour-free tongue tissue in patients with SCCOT we have identified 554 genes to be dysregulated compared to healthy tongue (15). Among these KRT36 was shown to be tongue-specific. KRT36 mRNA levels were significantly lower in clinically normal tissue adjacent to SCCOT tumours and KRT36 was one of the most progressively downregulated genes in SCCOT tumours. High KRT36 levels were observed in normal tongue from healthy volunteers and the intermediate levels in clinically normal tongue from SCCOT patients (15). Therefore, K36 was speculated to represent a potential marker of early neoplastic changes in the tongue and a representative indicator of field cancerization effects in OSCCs, where molecular changes can be seen in cells distant from the malignancy (16-18).

In the present study we aimed at mapping the expression pattern of K36 in a panel of human tissues with particular focus on normal and malignant SCCOT tissue from the mobile tongue. For this study, a specific K36 antibody was produced, affinity purified and used for immunohistochemical studies.

\section{Materials and methods}

Antibody production and purification. Two peptide sequences were chosen for antibody production; TPTFSTGSIKGLC from the N-terminus and CKPVIRVPSVPPV from the C-terminal region of human K36. These peptides were chosen based on their lack of sequence similarity with other human keratins using BLASTP search of the human proteome. The peptides were synthesised and individually coupled to keyhole limpet hemocyanin using their terminal cysteine residues, and antibodies were prepared in two rabbits using an equimolar mixture of the two peptide conjugates in each rabbit. Sera were collected after four immunisations and affinity purified against the individual peptides, to provide four separate affinity-purified rabbit K36 antibodies. Peptide synthesis, coupling, immunisation and affinity purification was performed commercially (Moravian Biotechnology).

Tissue specimens. A panel of morphologically normal tissue samples was collected for analysis of K36 expression. This panel comprised samples from different areas of the mobile tongue, excluding the base of the tongue, skin from different anatomical locations, lip, mamilla, nail, thymus, vaginal epithelium, cervix, appendix and oesophagus. The tumour tissue panel included skin cancers such as melanoma (1 case) and basal cell carcinoma (1 case), SCCOT (14 cases) and cervical squamous cancers (12 cases) (Table III). SCCOT samples were derived from the archive at Clinical Pathology between 2012 and 2017 from patients with primary SCCOT and sufficient material for immunohistochemical analysis. The total number of sections tested was 59 and out of these 28 were cancer samples. All tested samples of normal and cancer tissue were obtained from patients undergoing treatment in Umea and Brno. The present study was approved by the Ethical review boards in Umea and Brno (Dnr 08-003M; 03-201). Clinical information about the samples included are summarised in Tables I and II.

Immunohistochemistry. Immunohistochemical staining was performed on $4 \mu \mathrm{m}$ thick tissue sections and the optimal antibody concentration and retrieval were set. The procedure included section deparaffinization in xylene and rehydration into PBS through a graded series of ethanol. Endogenous peroxidase activity was quenched in $3 \%$ hydrogen peroxide in PBS for $5 \mathrm{~min}$. Antigen retrieval was performed by boiling in $1 \mathrm{mM}$ EDTA $\mathrm{pH} 8.0$ for $20 \mathrm{~min}$. Afterwards the sections were incubated overnight at $4^{\circ} \mathrm{C}$ with affinity purified rabbit serum CKP 39 fraction 1.4. HRP polymer conjugated anti-rabbit (K4003; Agilent Technologies, Inc.) was used according to the manufacturer's instructions (Envision + System; Agilent Technologies, Inc.). Signal was visualized by 3,3'-diaminobenzidine (Liquid DAB + substrate chromogen system; Agilent Technologies, Inc.). Nuclear counterstaining was performed with Gill's haematoxylin. Slides were graded as positive or negative.

Illumina HT-12 bead chip arraydata. The gene expression array data come from our previous study of SCCOT samples using Illumina HT-12 bead chip array containing 47,231 probes (15) that was enlarged by new samples. Here we retrieved and analysed mRNA levels of hair-keratins in data from 14 cases of normal control tongue from healthy volunteers, 22 SCCOT samples and 22 corresponding samples of tumour-free area of the tongue. Raw data has been deposited at http://www. ebi.ac.uk/arrayexpress, Array Express accession numbers E-MTAB-4678 (14) (Cases; all controls, tumour/tumour-free $11,35,51,56,58,59,61,65,73,79,85)$ and E-MTAB-5534 (Cases 20, 35, 49, 76, 98, 105, 111, 119, 124, 131, 137, 138).

Statistical analysis. Statistical analysis was performed on the previously published array data (14) using IBM SPSS 25 (IBM Corp.). Differences at $\mathrm{P} \leq 0.05$ were considered to be statistically significant. Changes in the level of expression of each $K R T$ gene within group of samples (control, tumour-free, tumour) was analysed using ANOVA with post hoc Tukey HSD test. Data are analysed as the mean \pm standard deviation. 
Table I. SCCOT clinical information.

\begin{tabular}{lll}
\hline Sex & Age (years) & \multicolumn{1}{c}{ TNM } \\
\hline F & 54 & T1N0M0 \\
M & 57 & T2N0M0 \\
F & 74 & T1N0M0 \\
M & 64 & T1N0M0 \\
F & 87 & T3N2cM0 \\
F & 74 & T2N0M0 \\
M & 67 & T2N0M0 \\
M & 55 & T4aN2bM0 \\
F & 74 & T2N0M0 \\
F & 71 & T2N0M0 \\
M & 51 & T2N1M0 \\
F & 71 & T1N0M0 \\
F & 42 & T1N1M0 \\
M & 52 & T4aN2bM0 \\
\hline
\end{tabular}

F, female; M, male; SCCOT, squamous cell carcinoma of the mobile tongue. TNM stage was allocated according to the 7 th edition of the AJCC cancer staging manual.

Table II. Clinical information of the additional samples studied.

\begin{tabular}{lccr}
\hline Tissue & Sex & $\begin{array}{c}\text { Mean age } \\
\text { (years) }\end{array}$ & $\begin{array}{r}\text { No. of } \\
\text { cases }\end{array}$ \\
\hline Appendix & F & 36 & 1 \\
Cervix & F & 50 & 3 \\
Hair, cross section & M & 10 & 1 \\
Hair, longitudinal section & F & 33 & 2 \\
Lip & F & 46 & 2 \\
Mammilla & F & 55 & 2 \\
Nail & F/M & $63 / 33$ & 2 \\
Oesophagus & F & 55 & 1 \\
Palmar skin & F & 82 & 2 \\
Skin of the scalp & M & 64 & 1 \\
Skin & $3 F, 2 M$ & $49 / 74$ & 5 \\
Thymus & F/M & $35 / 23$ & 2 \\
Tongue & F/M & $24 / 52$ & 6 \\
Vaginal epithelium & F & 45 & 1 \\
Basalioma & M & 42 & 1 \\
Cervical cancer & F & 47 & 12 \\
Melanoma & F & 73 & 1 \\
\hline
\end{tabular}

M, male; F, female.

Expression analysis. Microarray data were analysed using application Multiple Experiment Viewer (MeV) (19).

\section{Results}

Production of K36 antibodies. A mixture of two peptides from the human K36 protein (conjugated to keyhole-limpet hemocyanin) was used to immunise rabbits for antibody production. Sera were then used in dot-blots against the individual peptides (conjugated to bovine serum albumin) to assess antibody production. In both rabbits, a stronger response was seen against the CKPVIRVPSVPPV peptide (C-terminal region) than against the $\mathrm{N}$-terminal peptide (Fig. S1). Sera were then individually affinity-purified against each peptide and fractions were assessed for immunoglobulin purity and amount by polyacrylamide gel electrophoresis, again showing higher levels of antibodies to the $\mathrm{C}$-terminal region peptide in both rabbits (Fig. S2). These initial assessments were performed and provided by the supplier.

Affinity-purified antibodies and non-affinity purified sera were then tested at various concentrations on normal human tongue samples, employing antigen retrieval (EDTA or citrate) or no antigen retrieval. Pre-immune sera from the two rabbits were used as controls for their respective non-purified sera and affinity-purified reagents. From this initial screening, serum from rabbit 39 affinity-purified against the CKPVIRVPSVPPV peptide was chosen for further analysis, using antigen retrieval in $1 \mathrm{mM}$ EDTA boiling for $20 \mathrm{~min}$. Serum from rabbit 33 purified against the same peptide showed similar staining characteristics but required a lower dilution, whilst both sera purified against the TPTFSTGSIKGLC peptide showed similar staining but required high concentrations with attendant background staining. The non-immune sera did not show any staining when used at similar or higher concentrations.

Keratin 36 expression in normal tongue and SCCOT. Having established optimal conditions, we analysed K36 in the set of selected tissues using immunohistochemistry with affinity purified serum (designated 39-CKP). In view of our finding of altered KRT36 mRNA levels in SCCOT, we focused on staining K36 in normal and malignant human tongue epithelium.

No scoring of percentage K36 expressing cells or intensity in expression was performed. Tongue epithelium is a morphologically variable tissue with diverse structures. K36 immunostaining was localised solely to filiform papillae and not detected in other structures (Fig. 1). Within the filiform papilla, K36 was present in the cytoplasm of epithelial cells organised in arcs at the periphery. These cell clusters with elongated shape are called secondary filiform papillae and give rise to cornified spines (6). Other compartments of filiform papillae such as primary structure and interpapillary epithelium were K36-negative (Fig. 2).

We also analysed the distribution of K36-positive filiform papillae on the dorsal front tongue epithelium and found that all filiform papillae recognized in the anterior two-thirds of the mucosa were K36-positive.

To see whether K36 is dysregulated in cancer tissue, we also included samples of SCCOT, but K36 was not detected in any of these samples (Table III and Fig. 2).

Keratin 36 in various normal epithelial tissues and corresponding cancer tissue. We also used a panel of normal epithelial tissues chosen according to RNA sequencing data available in the Human protein atlas open access database (19). Based on these data and the literature, we compiled a set of human normal and cancer tissues that express KRT36 mRNA 
A

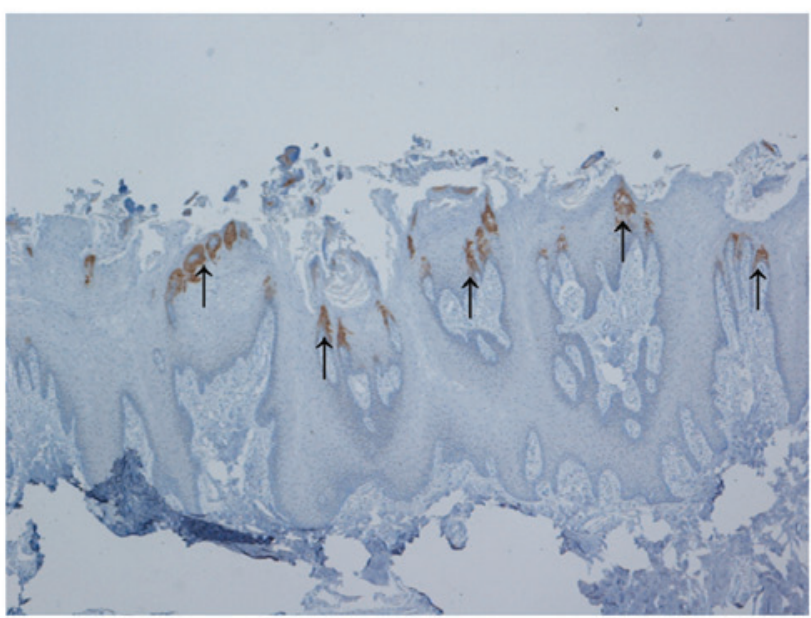

B

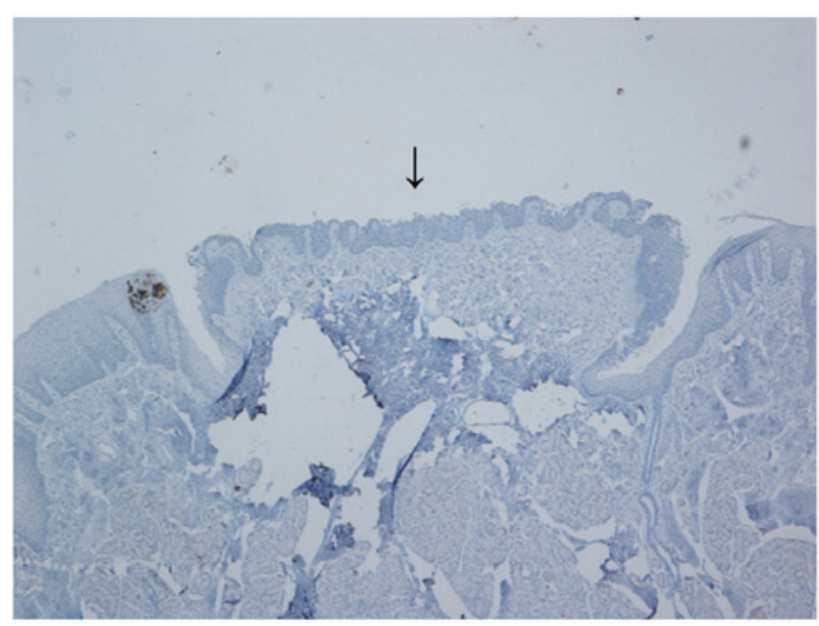

Figure 1. Immunohistochemical staining of dorsal tongue epithelium. K36 was positively expressed in the (A) filiform papillae (black arrows) and negatively expressed in the (B) papilla circumvalata (black arrows; magnification, $\mathrm{x} 40$ ). K36, keratin 36.

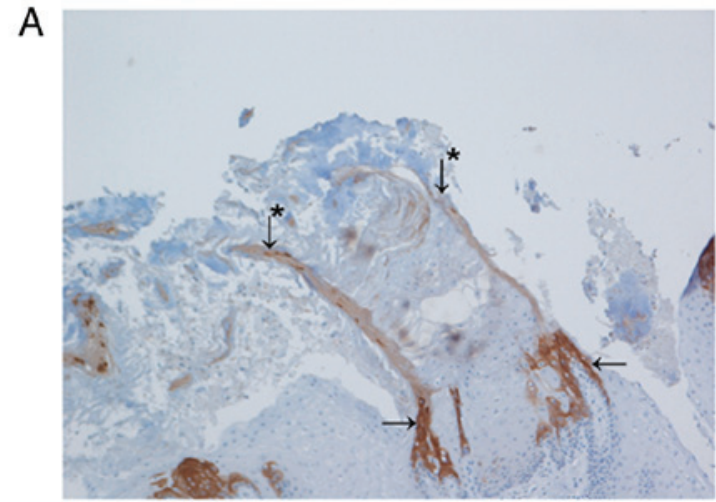

C

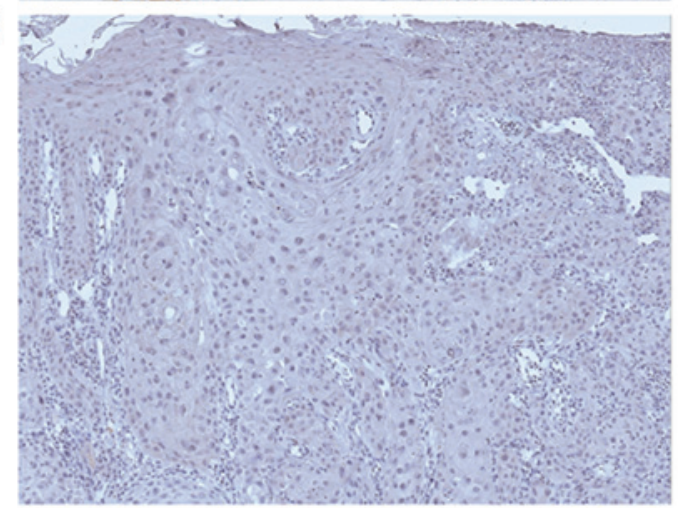

B

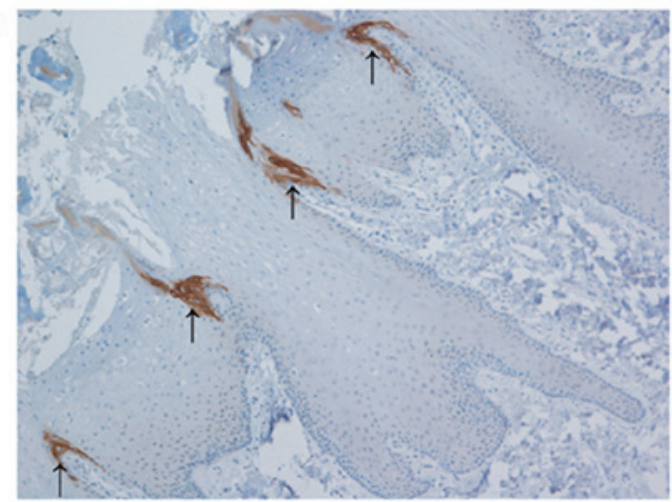

$\mathrm{D}$

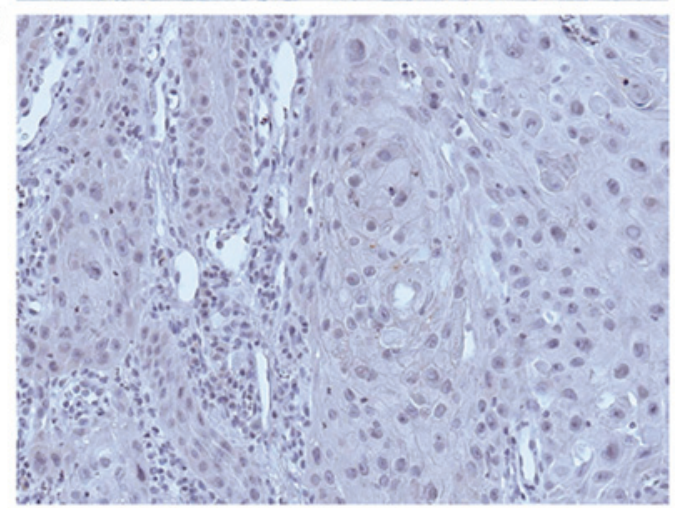

Figure 2. Staining and localisation of K36-positve cells within filiform papillae and SCCOT. (A) K36-positive cells (black arrows) and the elongation of cornified spines to the surface of dorsal tongue epithelium (asterisks) were observed (magnification, x100). (B) K36-positive cells (black arrows) were located at the periphery of the filiform papillae (magnification, x100). Tumour cells did not express K36 in SCCOT when observed at a magnification, (C) x100 and (D) $x 200$. SCCOT, squamous cell carcinoma of the oral tongue. K36, keratin 36.

but that were not yet evaluated at protein level. The panel of normal tissue samples included several positive cases, namely nail bed, Hassal's corpuscles of thymus and hair cortex (Fig. 3). Squamous epithelia from different anatomical locations, lip, mammilla, cervix, oesophagus and vagina were K36-negative and non-squamous epithelia in these tissues and appendix were also negative. Tumour samples of melanoma, basal cell carcinoma and cervical carcinoma were also negative for K36. An overview of staining results is provided in Table III.
Hair keratin RNA expression in tongue tumour, tumour-free and control samples. We analysed mRNA data from tongue tumours, tumour-free and healthy controls (14) concerning levels of individual hair type keratins (KRT31-KRT38 and KRT81-KRT86) in each group of samples (Fig. 4). Statistical analysis revealed significant downregulation of KRT3I $(\mathrm{P}=0.003)$, KRT33A $(\mathrm{P}=0.000)$, KRT33B $(\mathrm{P}=0.000)$, KRT34 $(\mathrm{P}=0.000)$, KRT36 $(\mathrm{P}=0.000)$, KRT84 $(\mathrm{P}=0.000)$ and KRT85 $(\mathrm{P}=0.000)$ in tumour samples compared to healthy controls. 
Table III. Overview of K36 antibody stained tissues.

\begin{tabular}{|c|c|c|c|}
\hline Tissue & No. of tested cases & Staining pattern & Localisation \\
\hline \multicolumn{4}{|l|}{ Normal tissue } \\
\hline Appendix & 1 & Negative & \\
\hline Cervix & 3 & Negative & \\
\hline Hair, cross section & 1 & Negative & \\
\hline Hair, longitudinal section & 2 & Positive & Cortex \\
\hline Lip & 2 & Negative & \\
\hline Mammilla & 2 & Negative & \\
\hline Nail & 2 & Positive & Nail bed \\
\hline Oesophagus & 1 & Negative & \\
\hline Palmar skin & 2 & Negative & \\
\hline Scalp skin & 1 & Negative & \\
\hline Skin other location & 5 & Negative & \\
\hline Thymus & 2 & Positive & Hassal's corpuscles \\
\hline Tongue & 6 & Positive & $\begin{array}{l}\text { Dorsal epithelium/filiform } \\
\text { papillae }\end{array}$ \\
\hline Vaginal epithelium & 1 & Negative & \\
\hline \multicolumn{4}{|l|}{ Cancer tissue } \\
\hline Basal cell carcinoma & 1 & Negative & \\
\hline Cervical squamous cancer & 12 & Negative & \\
\hline Melanoma & 1 & Negative & \\
\hline Tongue cancer & 14 & Negative & \\
\hline
\end{tabular}

Table summarises the number of tested samples and its specific localisation. K36, keratin 36.
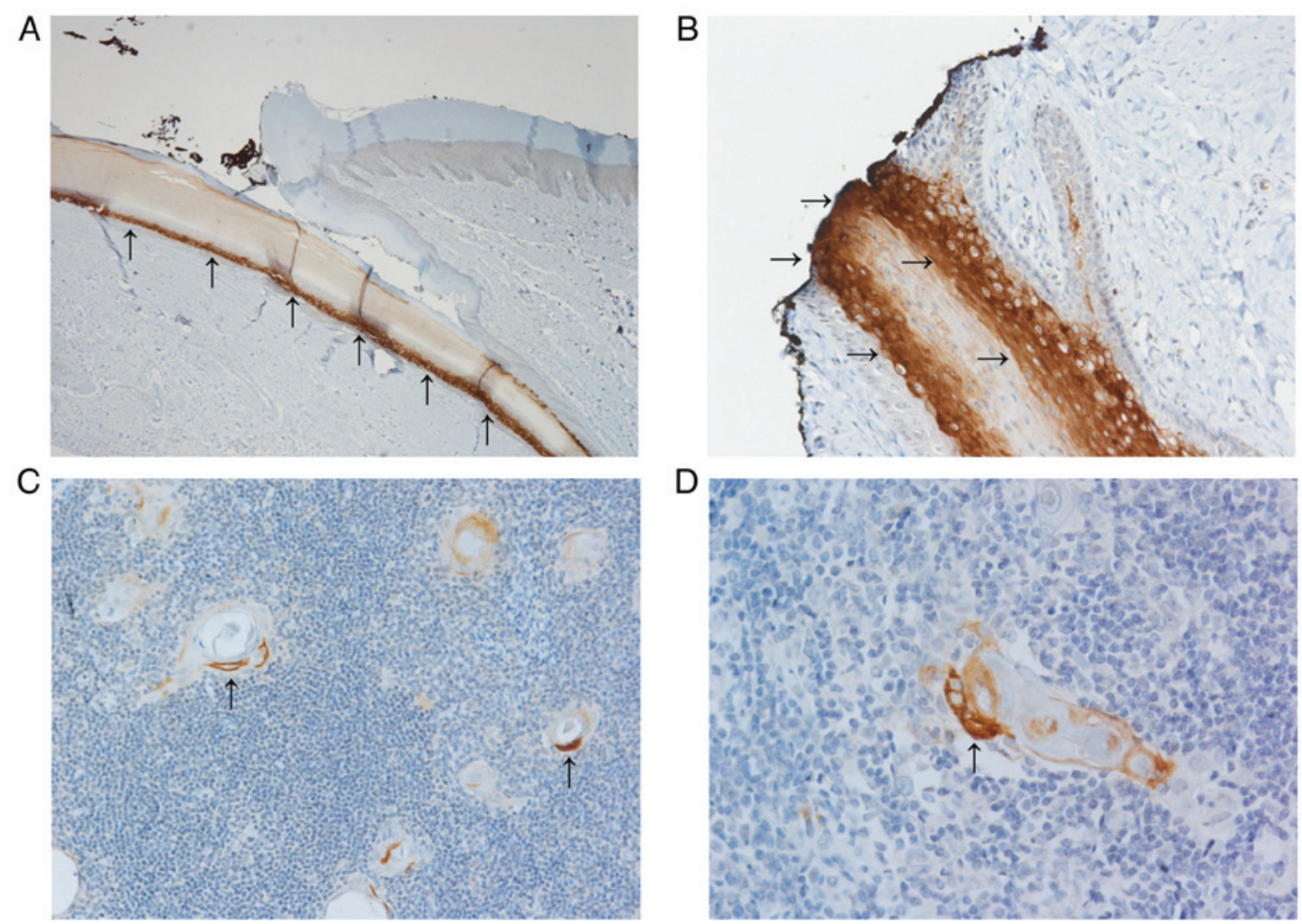

Figure 3. K36 expression in normal epithelial tissues. Immunohistochemical detection of K36 in the (A) nail bed (magnification, x40) and (B) nail papilla (magnification, x100), and Hassal's corpuscles. Images are presented at magnifications, (C) x100 and (D) x200. K36, keratin 36. 
A

\begin{tabular}{llr}
\hline 4.1779203 & 4.496567 & 11.335195
\end{tabular}

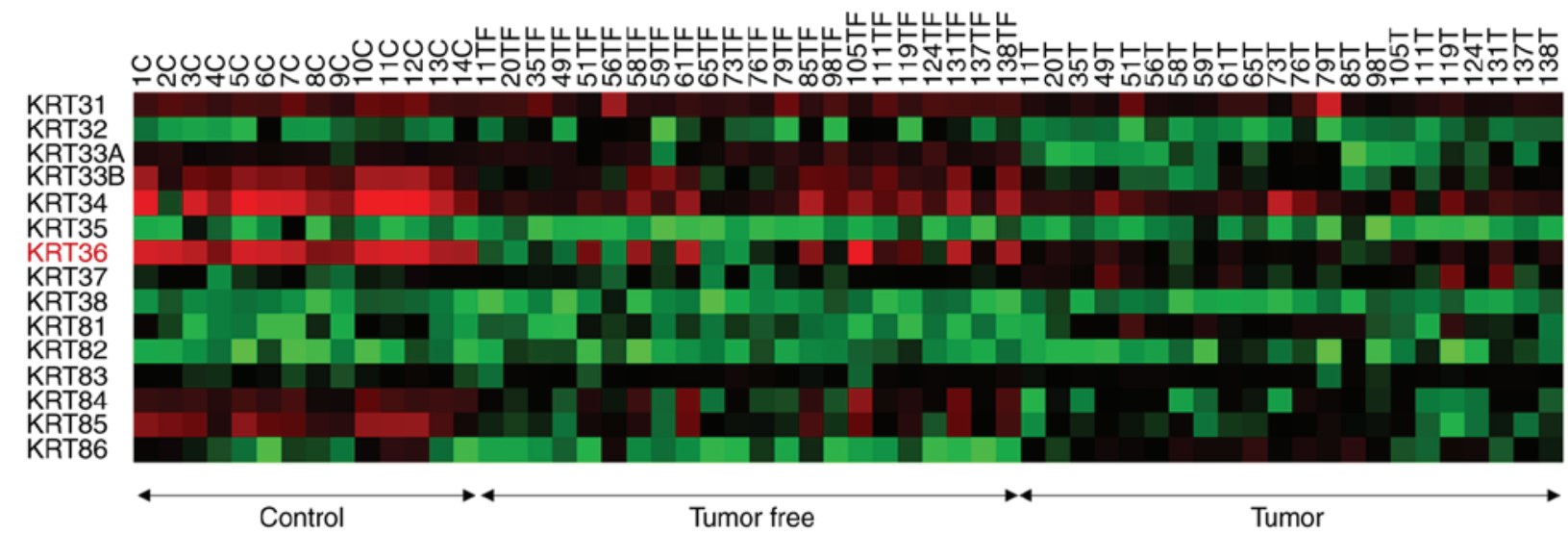

B

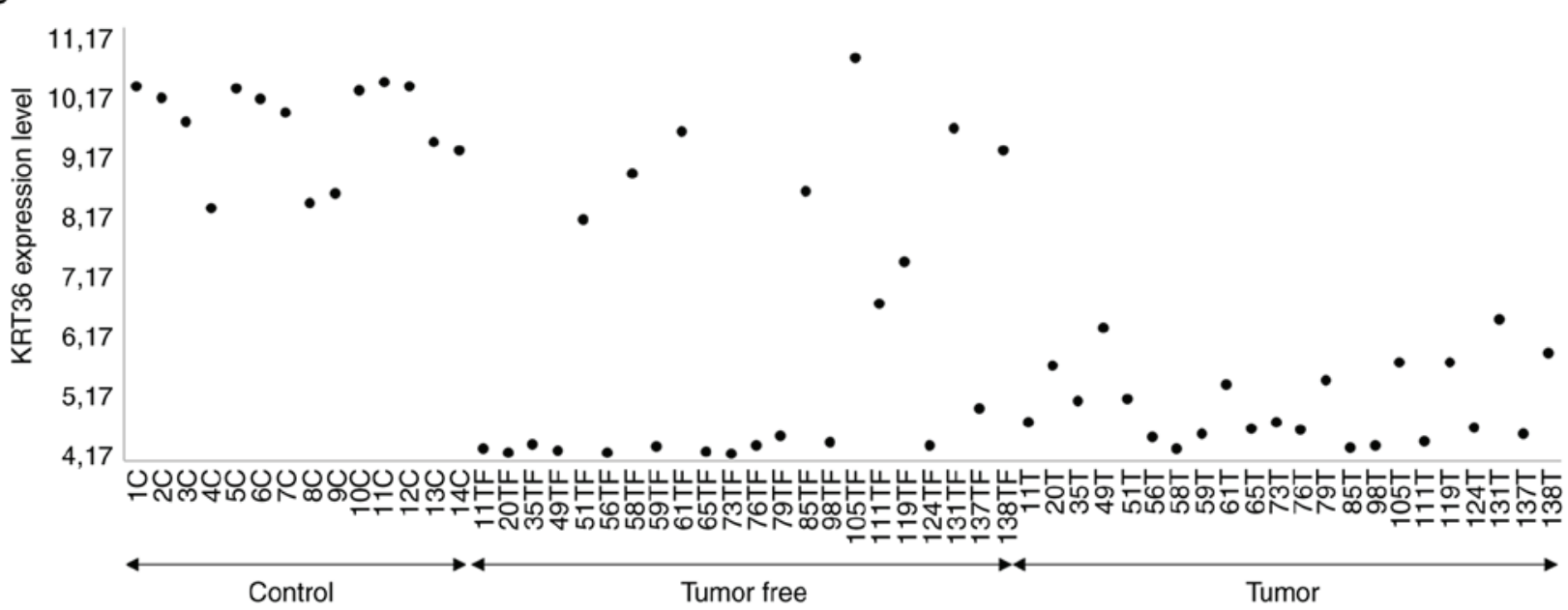

Figure 4. mRNA expression analysis of hair keratins. (A) Heat map demonstrated differences in mRNA expression between specific keratins in healthy controls, tumour free and tumour samples. (B) Overview of KRT36 expression in each sample within C, TF and T groups is graphically presented. C, healthy controls; TF, tumour-free; T, tumour.

Of these, KRT84 mRNA showed the highest correlation with KRT36 mRNA levels. Levels of KRT37 $(\mathrm{P}=0.000)$ and KRT81 $(\mathrm{P}=0.000)$ in contrast were significantly upregulated in cancer (ANOVA/Tukey HSD test) (Table SI and Fig. S3).

\section{Discussion}

Hair type keratins are expressed in hard keratinized structures such as hair and nails. Surprisingly, using a pan-specific antibody against hair keratins, it has been recognized that some hair keratins also occur in soft tissues such as mouse and human tongue (4). Specifically for K36, there is no record on protein expression up to now and mRNA data suggest it to be present in skin, thymus, oesophagus, spleen, vagina, testis (20), tongue epithelium and hair cortex (4). Here we investigated expression of the K36 protein using newly developed antibodies and found expression in thymus, tongue and hair only. In addition, we found K36 in nail beds. Most of these K36 expressing tissues are located in mechanically stressed regions of the human body. It has been stated that keratin filaments generally function as a major component of the epithelial cytoskeleton important for mechanical integrity of tissues (1) and thus we suggest that K36 may help to protect against mechanical stress. This is particularly in keeping with K36 as a component of filiform papillae which serve to provide a roughened surface of the dorsal tongue and also are important for providing traction during mastication. These structures therefore undergo extensive mechanical stress and must be firm as well as flexible.

Previously it has been described that Hassal's corpuscles of thymus display a very complex pattern of cytokeratins (CKs) with specific localisation within the structure. Hassal's corpuscles originate in medullary epithelial cells and resemble immature keratinocytes (21). Here K36 was mostly located in the intermediate to outer layers of cellular swirls, where CKs of simple epithelia such as CK7, 8, 18, 10, and CK4 and 17 from transitional epithelium are known to be located (21).

Our original data on mRNA expression profiling, showed that KRT36 mRNA was absent or levels very low in SCCOT, high in normal healthy tongue and intermediate in tumour-adjacent tongue. Here we focused on protein levels of $\mathrm{K} 36$ to see if this protein could be used as a diagnostic and/or prognostic marker in SCCOT. In accordance with our RNA data, we confirmed lack of K36 expression in all tumour 
samples. Even if the group of SCCOTs analysed, 14 cases, is limited, it is evident that KRT36 is downregulated in all tumours, whereas normal tongue tissue contains K36-positive cells. From the other tissue types selected from available RNA data, but not yet analysed at protein level, we also saw lack of $\mathrm{K} 36$ in both normal and tumourous cervical tissue. Also the single cases of basal cell carcinoma and melanoma included were devoid of K36.

It is unclear whether K36 downregulation is associated primarily with the tumour originating from a K36-negative population, or whether it comes with tumour progression or tumour dedifferentiation. As mechano-stimulation is rather unexplored in tumour biology, it can only be speculated that K36 downregulation in SCCOT can contribute to tumour progression by changing the mechanical phenotype of cancer cells.

In conclusion, using a novel affinity-purified polyclonal antibody we have identified K36 in several normal human tissues including filiform papillae of dorsal tongue epithelium, nail bed and Hassal's corpuscles, whereas other stratifying and non-stratifying epithelia were negative. In contrast, epithelial tumours like SCCOT and other squamous cell cancers were consistently negative for K36.

\section{Acknowledgements}

Not applicable.

\section{Funding}

This study was supported by grants from the Cancer Research Foundation in Northern Sweden, the Swedish Cancer Society Contract number 1805 42, Västerbotten County Council, Umeå University, European Regional Development Fund: Project ENOCH (grant no. CZ.02.1.01/0.0/0.0/16_019/0000868) and Ministry of Health in the Czech Republic (grant no. MMCI 00209805).

\section{Availability of data and materials}

The datasets used and/or analysed are available from the corresponding author on reasonable request.

\section{Authors' contributions}

PJC, BV, VB and $\mathrm{KN}$ designed the experiments, analysed the results and wrote the manuscript. VB, VH, LB, PF, NS performed the experiments, analysed the results and wrote the manuscript. All authors read and approved the final manuscript.

\section{Ethics approval and consent to participate}

The present study was approved by the local Ethical Review Boards of Umea and Brno (approval nos. Dnr 08-003M and 03-201).

\section{Patient consent for publication}

Not applicable.

\section{Competing interests}

BV is associated with Moravian Biotechnology, the company that produced and supplied the K36 polyclonal antibody. The company did not provide financial support for the present study and had no influence on the design, execution or analysis of the experiments. The other authors declare that they have no competing interests.

\section{References}

1. Ramms L, Fabris G, Windoffer R, Schwarz N, Springer R, Zhou C, Lazar J, Stiefel S, Hersch N, Schnakenberg U, et al: Keratins as the main component for the mechanical integrity of keratinocytes. Proc Natl Acad Sci USA 110: 18513-18518, 2013.

2. Schweizer J, Bowden PE, Coulombe PA, Langbein L, Lane EB, Magin TM, Maltais L, Omary MB, Parry DA, Rogers MA and Wright MW: New consensus nomenclature for mammalian keratins. J Cell Biol 174: 169-174, 2006.

3. Toivola DM, Strnad P, Habtezion A and Omary MB: Intermediate filaments take the heat as stress proteins. Trends Cell Biol 20: 79-91, 2010.

4. Dhouailly D, Xu C, Manabe M, Schermer A and Sun TT: Expression of hair-related keratins in a soft epithelium: Subpopulations of human and mouse dorsal tongue keratinocytes express keratin markers for hair-, skin- and esophageal-types of differentiation. Exp Cell Res 181: 141-158, 1989.

5. Heid HW, Moll I and Franke WW: Patterns of expression of trichocytic and epithelial cytokeratins in mammalian tissues. II. Concomitant and mutually exclusive synthesis of trichocytic and epithelial cytokeratins in diverse human and bovine tissues (hair follicle, nail bed and matrix, lingual papilla, thymic reticulum). Differentiation 37: 215-230, 1988.

6. Manabe M, Lim HW, Winzer M and Loomis CA: Architectural organization of filiform papillae in normal and black hairy tongue epithelium: Dissection of differentiation pathways in a complex human epithelium according to their patterns of keratin expression. Arch Dermatol 135: 177-181, 1999.

7. Rogers MA, Winter H, Langbein L, Bleiler R and Schweizer J: The human type I keratin gene family: Characterization of new hair follicle specific members and evaluation of the chromosome 17q21.2 gene domain. Differentiation 72: 527-540, 2004.

8. Tobiasch E, Winter H and Schweizer J: Structural features and sites of expression of a new murine 65 and $48 \mathrm{kD}$ hair-related keratin pair, associated with a special type of parakeratotic epithelial differentiation. Differentiation 50: 163-178, 1992.

9. Langbein L, Rogers MA, Winter H, Praetzel S, Beckhaus U, Rackwitz HR and Schweizer J: The catalog of human hair keratins. I. Expression of the nine type I members in the hair follicle. J Biol Chem 274: 19874-19884, 1999.

10. Langbein L, Rogers MA, Winter H, Praetzel S and Schweizer J: The catalog of human hair keratins. II. Expression of the six type II members in the hair follicle and the combined catalog of human type I and II keratins. J Biol Chem 276: 35123-35132, 2001.

11. Ng JH, Iyer NG, Tan MH and Edgren G: Changing epidemiology of oral squamous cell carcinoma of the tongue: A global study. Head Neck 39: 297-304, 2017.

12. Boldrup L, Coates PJ, Laurell G and Nylander K: Differences in p63 expression in SCCHN tumours of different sub-sites within the oral cavity. Oral Oncol 47: 861-865, 2011.

13. Boldrup L, Coates PJ, Wahlgren M, Laurell G and Nylander K: Sub-site based alterations in miR-21, miR-125b, and miR-203 in squamous cell carcinoma of the oral cavity and correlation to important target proteins. J Carcinog 11: 18, 2012.

14. Braakhuis BJ, Tabor MP, Kummer JA, Leemans CR and Brakenhoff RH: A genetic explanation of Slaughter's concept of field cancerization: Evidence and clinical implications. Cancer Res 63: 1727-1730, 2003.

15. Boldrup L, Gu X, Coates PJ, Norberg-Spaak L, Fahraeus R, Laurell G, Wilms T and Nylander K: Gene expression changes in tumor free tongue tissue adjacent to tongue squamous cell carcinoma. Oncotarget 8: 19389-19402, 2017.

16. Boldrup L, Coates PJ, Laurell G, Wilms T, Fahraeus R and Nylander K: Downregulation of miRNA-424: A sign of field cancerisation in clinically normal tongue adjacent to squamous cell carcinoma. Br J Cancer 112: 1760-1765, 2015. 
17. Leemans CR, Braakhuis BJ and Brakenhoff RH: The molecular biology of head and neck cancer. Nat Rev Cancer 11: 9-22, 2011.

18. Lochhead P, Chan AT, Nishihara R, Fuchs CS, Beck AH, Giovannucci E and Ogino S: Etiologic field effect: Reappraisal of the field effect concept in cancer predisposition and progression. Mod Pathol 28: 14-29, 2015.

19. Saeed AI, Sharov V, White J, Li J, Liang W, Bhagabati N, Braisted J, Klapa M, Currier T, Thiagarajan M, et al: TM4: A free, open-source system for microarray data management and analysis. Biotechniques 34: 374-378, 2003.

20. Ponten F, Jirstrom K and Uhlen M: The human protein Atlas - A tool for pathology. J Pathol 216: 387-393, 2008.
21. Shezen E, Okon E, Ben-Hur H and Abramsky O: Cytokeratin expression in human thymus: Immunohistochemical mapping. Cell Tissue Res 279: 221-231, 1995.

(c) (1) $(9$ This work is licensed under a Creative Commons Attribution-NonCommercial-NoDerivatives 4.0 International (CC BY-NC-ND 4.0) License. 